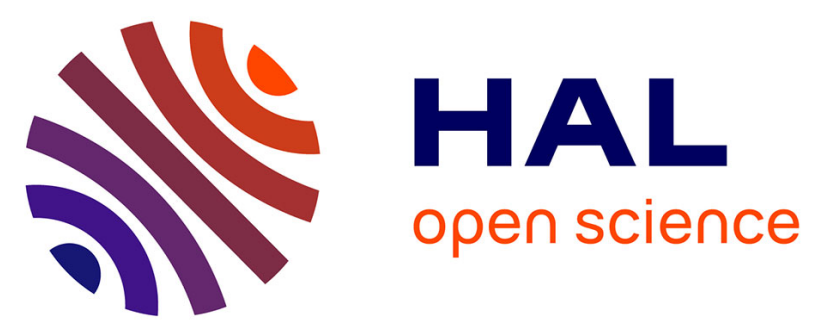

\title{
A worldwide and annotated database of evaporative water loss rates in squamate reptiles
}

Jean-François Le Galliard, Chloé Chabaud, Denis Otávio Vieira de Andrade, Francois Brischoux, Miguel Angel Carretero, Andréaz Dupoué, Rodrigo S. B. Gavira, Olivier Lourdais, Marco Sannolo, Tom JM Van Dooren

\section{To cite this version:}

Jean-François Le Galliard, Chloé Chabaud, Denis Otávio Vieira de Andrade, Francois Brischoux, Miguel Angel Carretero, et al.. A worldwide and annotated database of evaporative water loss rates in squamate reptiles. Global Ecology and Biogeography, 2021, 30 (10), pp.1938-1950. 10.1111/geb.13355 . hal-03301335

\section{HAL Id: hal-03301335 https://hal.science/hal-03301335}

Submitted on 29 Jul 2021

HAL is a multi-disciplinary open access archive for the deposit and dissemination of scientific research documents, whether they are published or not. The documents may come from teaching and research institutions in France or abroad, or from public or private research centers.
L'archive ouverte pluridisciplinaire HAL, est destinée au dépôt et à la diffusion de documents scientifiques de niveau recherche, publiés ou non, émanant des établissements d'enseignement et de recherche français ou étrangers, des laboratoires publics ou privés. 


\section{A worldwide and annotated database of evaporative water loss rates in}

\section{2 squamate reptiles}

3 Jean-François Le Galliard*1,2, Chloé Chabaud ${ }^{1,3}$, Denis Otávio Vieira de Andrade ${ }^{4}$, François

4 Brischoux $^{3}$, Miguel A. Carretero ${ }^{5,6}$, Andréaz Dupoué ${ }^{1,2}$, Rodrigo S. B. Gavira ${ }^{3}$, Olivier Lourdais ${ }^{3}$,

5 Marco Sannolo $^{5,7}$ and Tom J. M. Van Dooren ${ }^{1}$

6 1. iEES Paris, Sorbonne Université, CNRS, UMR 7618, Tours 44-45, 4 place Jussieu, 75005, Paris, France

7 2. Ecole normale supérieure, PSL Research University, Département de biologie, CNRS, UMS 3194, Centre

8 de recherche en écologie expérimentale et prédictive (CEREEP-Ecotron IleDeFrance), 78 rue du château,

977140 Saint-Pierre-lès-Nemours, France

10 3. Centre d'Etudes Biologiques de Chizé - ULR CNRS UMR 7372, 79360 Villiers en Bois, France

11 4. Departamento de Zoologia, Instituto de Biociências, Universidade Estadual Paulista - UNESP, Bela Vista

12 CEP 13506-900, Rio Claro, Brasil

13 5. CIBIO Centro de Investigação em Biodiversidade e Recursos Genéticos, InBIO, Universidade do Porto,

14 Campus de Vairão, Rua Padre Armando Quintas, N 7. 4485-661 Vairão, Vila do Conde, Portugal

15 6. Departamento de Biologia, Faculdade de Ciências da Universidade do Porto, R. Campo Alegre, s/n, 4169 -

16 007, Porto, Portugal

177 Department of Evolutionary Ecology, Museo Nacional de Ciencias Naturales - CSIC, C/José Gutiérrez

18 Abascal 2, 28006, Madrid, Spain

19

20 * Correspondence: Jean-François Le Galliard

22 Running head: SquamEWL: a database of evaporative water loss in reptiles

\section{Biosketch}


25 Jean-François Le Galliard is an evolutionary biologists and population ecologist interested in the

26 understanding the diversity of life history and behavioral strategies that animals use to survive and

27 reproduce in their changing environment. For this data project, he worked with a team of ecologists

28 and evolutionary biologists with strong expertise in the ecophysiology and water biology of reptiles.

\section{Authors contributions}

31 JFLG and TVD conceived the project, organized the data collection, and collected and checked

32 data. FB, AD, RG and OL contributed to project conception and helped with data collection and

33 management. MA, MS and DOVA contributed data and helped with data collection. CC helped with

34 data collection, data formatting and technical validation. All authors contributed to writing based on

35 a first version produced by JFLG.

\section{Acknowledgments}

38 We thank colleagues who helped with data calculation by providing raw or summary data, metadata

39 or additional information including David Chapple, Allen Cohen, Don Bradshaw, David Chapple,

40 Pierre-André Crochet, Sebastian Kirchhof, Jason Kolbe, Michaël Guillon, Shu-Ping Huang, Jason

41 Kolbe, Amy MacLeo, Bill Mautz, Francisco Javier Muñoz Nolasco, Anil Oguz, Panayiotis Pafilis,

42 Catarina Rato, Abderrahim S'khifa, Graham Thompson, Miguel Vences, Philip Withers and

43 Anamarija Žagar. We thank Luis Miguel Senzano for providing unpublished data. We thank two

44 anonymous reviewers and Michaël Kearney for comments that helped improve the manuscript.

$46 \quad$ Funding

47 Compilation of the database was funded by the Centre National de la Recherche Scientifique

48 (CNRS), the Agence Nationale de la Recherche (Aquatherm: ANR-17-CE02-0013 to JFLG) and a

49 doctoral grant from Ecole normale supérieure to CC. 


\section{Abstract}

51 Motivation: The understanding of physiological adaptations, of evolutionary radiations and of

52 ecological responses to global change urges for global, comprehensive databases of the functional

53 traits of extant organisms. The ability to maintain an adequate water balance is a critical functional

54 property influencing the resilience of animal species to climate variation. In terrestrial or semi-

55 terrestrial organisms, total water loss includes a significant contribution of evaporative water loss

56 (EWL). The analysis of geographic and phylogenetic variation in EWL rates must however account

57 for differences in methods and potential confounding factors which influence standard measures of

58 whole-organism water loss. We compiled the global and standardized SquamEWL database of total, 59 respiratory and cutaneous EWL for 325 species and sub-species of squamate reptiles (793 samples

60 and 2536 estimates) from across the globe. An extensive set of companion data and annotations

61 associated to the EWL measurements of potential value for future investigation, including

62 metabolic rate data, is provided. We present preliminary descriptive statistics for the compiled data,

63 discuss gaps and biases, and identify promising avenues to update, expand and explore this

64 database.

65 Main types of variables contained: standard water loss rates, geographic data, metabolic rates.

66 Spatial location: global.

67 Time period: data were obtained from extant species and were collected between 1945 and 2020.

68 Major taxa: Reptilia, Squamata including lizards, snakes and amphisbaenians.

69 Level of measurements: individual samples of animals from the same species, locality, age class

70 and sex category.

71 Software format: csv.

72 Keywords: ectotherms, functional traits, homeostasis, hydroregulation, macrophysiology,

73 evaporative water loss. 


\section{1. Background and summary}

75 The ecological niche of a species is an important concept in correlative species distribution modeling and describes the multivariate environmental space of abiotic and biotic factors that determine the boundaries of a species range (Chase \& Leibold, 2003). In mechanistic species distribution models, however, the ecological niche of a species is not inferred from its realized niche but derived from a calculation of the fundamental niche of the organism, defined as the full range of conditions and resources suitable for survival and reproduction (Kearney \& Porter, 2009). In the last decade, mechanistic models have become central to uncover the potential effects of global climatic change on species viability and distribution (Sinervo et al., 2010; Boyle et al., 2020). Since properties of the fundamental niche are determined by organismal traits, current research aims to better understand how morphological, physiological and behavioral properties of organisms constrain their fundamental niche and ultimately define their ecological niche and distribution. physiological, phenological or behavioral traits that determine the performance of individuals (Violle et al., 2007; Kearney et al., 2021), is a fundamental step in this research program (Schneider et al., 2019). Furthermore, global databases of functional traits can be used to investigate universal scaling rules and advance our understanding of evolutionary processes (Díaz et al., 2016; Etard et $a l ., 2020)$. Research on the climatic tolerances of ectothermic animals over the last decades has focused on the study of functional traits characterizing the thermal biology, including thermal limits

93 (Sunday et al., 2012; Bennett et al., 2018), thermal performance curves and metabolism (Dillon et

94 al., 2010), thermoregulation behavior (Kearney et al., 2009), or thermal sensitivity of development

95 (Noble et al., 2018). However, studies have shown that traits associated with water balance in

96 ectotherms are also critical in setting their climatic niche, their sensitivity to global changes, and

97 their macro-evolutionary radiation patterns (Brischoux et al., 2012; Kearney et al., 2018; Garcia- 
Porta et al., 2019; Gouveia et al., 2019; Rozen-Rechels et al., 2019; Lertzman-Lepofsky et al.,

99 2020).

In animals, water balance is dynamically regulated by the constant adjustments of water loss and water intake processes (Figure 1A) which depend on morphological features and physiological and behavioral mechanisms (e.g., behavioral hydroregulation, skin resistance to water loss or respiration, Chown et al., 2011; Pintor et al., 2016; Pirtle et al., 2019; Riddell et al., 2019). As a result, body hydration state is homeostatically maintained within a safety zone by compensating water loss with input from metabolic, food and drinking water to avoid the acute and chronic, potentially lethal, effects of dehydration. Total evaporative loss (TEWL) comprises both the water lost through the skin epidermis or exoskeleton (cutaneous water loss, CWL) and via the respiratory system (respiratory water loss, RWL). Although functional traits such as desiccation resistance and behavioral traits are also important for hydroregulation, the standard rate of EWL has proven to be a relevant metric to assess water regulation strategies, and to scale the susceptibility of organisms to drought, habitat aridity or salinity in birds (Albright et al., 2017; Boyle et al., 2020), mammals (Van

112 Sant et al., 2012), non-avian reptiles (Brischoux et al., 2012; Cox \& Cox, 2015), amphibians

113 (Lertzman-Lepofsky et al., 2020) and insects (Addo-Bediako et al., 2001). However, to our

114 knowledge, there has been no attempt to compile and annotate a global database of all published

115 EWL records in terrestrial animals, including those of squamate reptiles. Squamate reptiles share proximate mechanisms of water loss (Mautz, 1982). They are ideal model systems to study the relevance of water regulation strategies under a macroecological mechanistic approach since these dry-skinned ectothermic organisms exhibit great phylogenetic and ecological diversification, broad variation in body size and shape, and are found in most habitats

120 across the globe (Meiri, 2018). In addition, their performance and life history strategies are greatly

121 influenced by the availability of water in their environment (Kearney \& Porter, 2004; Lillywhite, 122 2017; Rozen-Rechels et al., 2020). Rates of TEWL vary with micro-climatic conditions, 
123 morphological and functional adaptations, life stages and behavioral strategies (e.g., space use and

124 activity, Pirtle et al., 2019). In squamates, EWL is not as tightly associated with body temperature

125 regulation as in endothermic animals. Indeed, heat loss due to CWL and RWL, i.e., evaporative

126 cooling, is generally negligible in squamate reptiles, except under extreme conditions such as

127 panting in some desert species or under extreme heat stress (Tattersall et al., 2006; Loughran \&

128 Wolf, 2020). Although CWL is generally the dominant avenue of water loss in squamate reptiles,

129 the partitioning between RWL and CWL, which includes trans-epidermal, ocular and cloacal water 130 loss, varies between and within species (Mautz, 1982; Pirtle et al., 2019). One important factor

131 affecting the partitioning between CWL and RWL is body size, which is largely determined by the

132 fact that surface area and skin thickness (the primary determinants of CWL) scale differently with

133 body size than respiration rate and lung size (primary determinants of RWL, Mautz, 1980, 1982) do.

134 Changes in patterns and rates of EWL can rapidly evolve in squamates, and there is evidence of 135 adaptive plastic responses of TEWL to fluctuating temperatures or hydric conditions (Kattan \& 136 Lillywhite, 1989; Moen et al., 2005; Cox \& Cox, 2015; Garcia-Porta et al., 2019; Sannolo et al., 137 2020). Yet, estimates of TEWL are influenced by methodological choices, sampling methods (e.g., 138 size class or seasonal factors), acclimation procedures and statistical reporting methods, which 139 makes comparisons across studies difficult without an unambiguously defined vocabulary and a 140 broad set of standardized metadata (see Figure 1B).

141 Here, we assembled a global and annotated database of rates of evaporative water loss in 142 squamate reptiles of the world using published information and unpublished data we collected in 143 recent years. Our initiative differs from previously published data sets by (1) its broad taxonomic 144 scope spanning all available data for all squamate reptiles including lizards, snakes and 145 amphisbaenians, (2) its exhaustiveness since we gathered all identified published estimates from a 146 pre-established list of acceptable methodologies and recorded samples and units from the original 147 publications without a priori exclusion of particular data or ad hoc calculations of statistics, and (3) 
148 its comprehensiveness since we compiled information on 30 metadata variables. We provide a

149 computing script to facilitate future use of these data. Whenever available, we recovered and report

150 all the variables necessary for the calculation of skin resistance to evaporation $R_{s}$, a functional trait

151 relevant to the predictive modeling of water-flow balance (Kearney et al., 2021) and of central

152 relevance in mechanistic niche distribution models (Riddell et al., 2017). We also compiled a

153 second companion database on metabolic rate $\left(\mathrm{VO}_{2}\right.$ and $\left.\mathrm{VCO}_{2}\right)$ for those cases in which this

154 information was also available on the same source material examined for the construction of the

155 EWL database. We judge this companion database relevant under the primary goal of this paper,

156 since it may ease future studies focusing on the partitioning between CWL and RWL (Gates, 1980;

157 Pirtle et al., 2019).

158 We make the compiled database freely available to stimulate future research on water

159 balance in reptiles, particularly on water conservation mechanisms and even more so on the

160 geographic, ecological, and phylogenetic correlates of evaporative water loss. We expect to

161 encourage other researchers to work on the expansion of the database and consult it to identify

162 understudied groups and which set of variables are relevant to be measured and reported. Finally,

163 we hope that our database will be useful for those willing to use it to construct mechanistic niche

164 models (Kearney \& Porter, 2020)

\section{2. Methods and dataset}

166 We searched for published literature and referenced reports providing potential data on water loss rates in squamate reptiles in Web of Science and Google Scholar using relevant search terms in title, abstract and content with the following query: ("water loss" OR "water balance" OR "hydroregulation") AND ("reptile*” OR "snake*” OR "lizard*” OR “squamate*”). In addition, we

170 extracted all references from a recent comparative analysis of water loss in reptiles (Cox \& Cox, 171 2015). The availability of water loss data on samples of individuals (excluding eggs or embryos) 
172 from known reptile species was then checked by a single person (JFLG) who stored all such

173 references, source files as well as available online data in a Zotero group library (see

$174 \mathrm{https}: / /$ tinyurl.com/y2nclru5). Using tags, all publications were then assigned to a single person who

175 oversaw confirming availability of water loss data, extracting the data and adding relevant metadata

176 to a spreadsheet. If additional relevant publications were identified, those were added to the Zotero

177 library and processed by the same individual. This procedure was performed first in September

1782018 and repeated in October 2019, February 2020, and September 2020 and spans data sources

179 published from 1932 to late 2020. Additionally, unpublished data were also contributed by our

180 research group. In March 2021, we added metadata following up on review requests, extracted

181 additional information on metabolism and validated the database again.

182 We produced a library of 160 publications, reports or academic contributions (monographs,

183 dissertations and theses) from which we extracted complete or partial data (see PRISMA workflow

184 in Supplementary File 1). EWL data were then added into a spreadsheet together with all the

185 available metadata describing the relevant conditions of water loss measurements, species and

186 sample characteristics, and contextual information regarding animal morphology, location, sampling

187 dates and habitat. The species and subspecies identities were standardized using the EMBL/EBI

188 Reptile DataBase release of December 21, 2019 (Uetz \& Etzold, 1996). We performed data

189 extraction accepting a sample data point as defined by a unique group of animals composed of a

190 fixed set of individuals, subjected to the same experimental protocol, and measured under the same

191 conditions. For each sample, we extracted the mean and dispersion statistics (SD, SE or range) of

192 EWL rates (total water loss, respiratory water loss or cutaneous water loss) and the mean and

193 dispersion statistics (SD, SE, or range) of body mass as well as mean statistics for body size (snout

194 to vent and total length) and body surface area. Data were taken from published or shared datasets,

195 extracted from tables and text, or extracted from figures using scanned images of the plots and the

196 Plot Digitizer program in Java (https://sourceforge.net/projects/plotdigitizer/). Information on 
measurement method, temperature (air, skin surface and core body temperature), water vapor pressure deficit (VPD) in the air, air flow, measurement duration, and activity statuses of animals were extracted whenever available or requested from the corresponding authors. We added additional information on chamber characteristics and average diameter of the chambers to make it possible to calculate air velocity from air flow. All data is presented as values on the scales chosen for reporting, although, whenever possible, the possibility for scale conversion is provided (see below). These metadata were selected because they provide important contextual information about dominant factors of methodological variation in measurements of EWL (Mautz, 1980).

A mechanistic understanding of water balance requires a careful quantification of the functional traits governing variation in CWL and RWL among species and the use of biophysical models of EWL (Gates, 1980; Kearney \& Porter, 2009; Pirtle et al., 2019). In RWL, water is lost by evaporation from the lung respiratory surface to air contained in it at a rate determined by the water vapor density gradient. The establishment of the water vapor density gradient, in turn, depends on the relative humidity and temperature of the inhaled air and the pace at which the air inside the lungs is renewed, i.e., total ventilation (Gates, 1980). Total ventilation is determined by a combination of breathing frequency and tidal volume and is linked to the adequate match of metabolic demand (Pirtle et al., 2019). Thus, RWL should increase monotonically with air temperature, air dryness, body temperature, and increased metabolic activity. CWL is mostly determined by the water vapor gradient between the skin surface and the air, which is established by the interaction of air temperature and relative humidity, air convection rate, an animal's shape and size, both affecting skin surface area, and, finally, the intrinsic resistance of the skin to evaporative water loss or $R_{s}$ (Gates, 1980; Mautz, 1980). Thus, from a functional standpoint, $R_{s}$ informs on a central organismal determinant of the animal's susceptibility to lose water via evaporation being, possibly, the best standardized metric to compare samples, populations and species (Gates, 1980; Pirtle et al., 2019). Several studies have quantified and compared $R_{s}$ among closely related 
squamate reptile species (Dmi'el, 1998, 2001; Oufiero \& Van Sant, 2018), but accurate values of $R_{s}$

223 are generally unavailable for most species to date (Mautz, 1982). Therefore, for those cases in

224 which the information could be recovered, we collected and reported all the components necessary

225 for calculating $R_{s}$, even though we have not added it as a singled-out variable in the dataset

226 (Kearney \& Porter, 2020). Note that there are potential caveats with respect to this approach

227 because of unknowns in the regional variation of skin and body temperatures (Barroso et al., 2016),

228 difficulties to partition sub-components of EWL (Senzano \& Andrade, 2018), and further study or

229 species-specific features (Mautz, 1982).

230 We also included variables describing study design (groups and treatments) and a quality

231 score permitting the exclusion of data (abnormal animals, animals maintained under manipulated

232 conditions or measurements performed in non-standard conditions) in future extraction and

233 analysis. Each of us scored a study data quality ranging from high (appropriate protocols, protocols

234 are well reported, and data statistics are detailed), medium (one item is missing) to low (poorly

235 designed, poorly reported protocols and poorly detailed data statistics). The same person also scored

236 the data standards as either usable to not usable data (because of low study standards or

237 inappropriate metadata, see detailed comments available in a free text item). The content of the

238 database is outlined in Table 1, which provides additional information on each field of the data

239 table. Upon data extraction, each record was checked by the data collectors and the content and

240 integrity of the whole database was checked by two individuals prior to uploading the first version,

241 called SquamEWL, in a public repository available at DOI: 10.5281/zenodo.3666172 (Le Galliard

242 et al., 2020). Routines for data extraction, database integrity check and data cleaning were coded in

243 the R statistical language, and are briefly described below. The fully annotated code written for R

244 version 3.6.3 (R Core Team, 2020) is also available in the public repository and includes functions

245 to convert records between measurement scales. 


\section{Preliminary analyses}

247 The SquamEWL dataset includes 2536 water loss records of 325 species and sub-species (301

248 unique species) of squamate reptiles for 793 unique samples (mean number of individuals per

249 sample $=10.15 \pm 11.5 \mathrm{SD}$, median $=6$, range=1-169) with most measurements obtained for TEWL

$250(\mathrm{~N}=2146)$ and substantially fewer for CWL (264) and RWL (126). The vast majority of records is

251 from field-captured animals $(\mathrm{N}=2015)$ in comparison to laboratory acclimated animals or those

252 raised in outdoor enclosures $(\mathrm{N}=203)$. There is substantial variation in the acclimation time of

253 animals (time spent in the laboratory prior to measurement), even after excluding laboratory-raised

254 animals (range $=0-750$ days, mean $=46.45 \pm 119.4 \mathrm{SD}$, median $=7$ ). The predominant protocol

255 involves measurements of body mass loss in the laboratory $(\mathrm{N}=1391)$, followed by direct

256 measurements of water vapor changes in flow-through chambers $(\mathrm{N}=760)$, measurements of the

257 mass increase of a desiccant $(\mathrm{N}=296)$, doubly-labeled water techniques in active animals $(\mathrm{N}=56)$

258 and, in more recent studies, flux chamber protocols for measurements of trans-epidermal water loss

$259(\mathrm{~N}=33)$. The variation in micro-climatic environmental conditions during sampling is wide with air 260 temperatures ranging from 5.3 to $45^{\circ} \mathrm{C}($ mean $=28 \pm 6.47 \mathrm{SD}$, median $=27)$, VPD in the air ranging 261 from ca. zero to $9.10 \mathrm{kPa}($ mean $=2.83 \pm 1.73 \mathrm{SD}$, median $=2.53)$ and air flow ranging from zero to 262 several hundred $\mathrm{mL}$ per $\min ($ mean $=146.5 \pm 267.8 \mathrm{SD}$, about half of the records were obtained in 263 still air).

The geographic origin is available for 1923 records comprising 316 unique localities (Figure 2A) predominantly located in Northern and Central America, Europe and Australia, with underrepresentation in pan-tropical diversity hotspots including South America, Africa and Asia as well as several semi-arid and arid regions of Africa, the Arabian Peninsula and remaining Asia subtropical regions (Roll et al., 2017). The altitudinal range varied from sea level up to 3,718 $\mathrm{m}$ above sea level with most records below $500 \mathrm{~m}($ mean $=367.7 \mathrm{~m}$, median $=74 \mathrm{~m})$, which reflects the 
prevalent altitudinal range for Squamata (Buckley et al., 2008, 2012). The dataset contains

271 representatives of 34 families but only $2.71 \%$ of the total species richness estimated for squamates.

272 Given the contribution of different families to the total species richness of squamate reptiles

273 worldwide (Uetz \& Etzold, 1996; Roll et al., 2017), there is an "over-representation" of species

274 from Lacertidae, Phrynosomatidae, Teiidae, Diplodactylidae, Sphaerodactylidae and Viperidae and

275 an "under-representation" of species from Gekkonidae, Gymnophthalmidae, Elapidae, Scincidae

276 and Colubridae (Figure 2B).

In addition to heterogeneity in sampling, measurements conditions and laboratory methods,

278 calculations of water loss rates vary among studies. In particular, EWL rates are reported on three

279 different scales, namely as mass-relative values (\% of initial body mass or mg per g per hour, 1544

280 records), absolute values (mg water per hour, 715 records), or surface-relative values (mg per $\mathrm{cm}^{2}$

281 per hour, 270 records). To convert all EWL records to a single scale (mg per hour), we gathered

282 records reported for the same samples under the same conditions on different scales and used a

283 statistical procedure to fit a calibration function to convert data from relative to absolute scale (see

284 Supplementary File 1). Using this approach, we calculated 1884 unique estimates of absolute EWL

285 rates, including TEWL, CWL and RWL components. Preliminary non-phylogenetic analyses

286 indicated that TEWL and CWL rates scale allometrically with body mass with an exponent of ca.

287 0.6-0.7 (linear regression on a log-log scale, TEWL: slope $=0.66 \pm 0.0154$ SE, CWL: slope $=0.59$

$288 \pm 0.048 \mathrm{SE}$; see Figure 2C), which is close to the $2 / 3$ allometric exponent for the geometric

289 increment of surface area with the increment in body mass. The RWL component is generally

290 smaller than CWL component of TEWL in small-sized species and increases faster with body mass

291 (linear regression on a log-log scale, RWL: slope $=0.88 \pm 0.058 \mathrm{SE}$ ), as expected from the $3 / 4$

292 allometric exponent relating ventilation and metabolic rates to body mass. After correction for body

293 mass but not for experimental conditions, records of TEWL showed no clear altitudinal cline across

294 all samples (linear regression with log-transformed WL and mass, $\mathrm{F}_{1,959}=0.56, \mathrm{P}=0.09$ ), but a 
small negative latitudinal cline (linear regression with log-transformed WL and mass, $\mathrm{F}_{1,960}=5.25, \mathrm{P}$

$296=0.02$ ). Future analyses should explore more in depth the effects of species characteristics and environmental conditions (e.g., temperature and humidity, habitat aridity) on inter-specific variation 298 in EWL.

Physical equations of TEWL require additional information on the temperature of the animals, the body shape and air velocity, and the metabolism (see above). Surface $(\mathrm{N}=90)$ and body $(\mathrm{N}=190)$ temperatures were very rarely reported because they are usually difficult to measure and it is often assumed that they are at equilibrium with air temperature, which is not true in general (Warburg, 1965). Air velocity could be calculated for most records $(\mathrm{N}=2204)$ and ranged from zero to $0.017 \mathrm{~m}$ per second $($ mean $=0.0014 \pm 0.0029 \mathrm{SD})$. We extracted 394 usable metabolic data values from the same sources as in SquamEWL database including 105 unique species and subspecies from 184 samples, which we present in a separate companion dataset. Notwithstanding that, we provide the necessary functions to merge these data with those of the SquamEWL for users potentially interested in mechanistic modeling of CWL and RWL using the NicheMapR software (Pirtle et al., 2019).

\section{0}

\section{Conclusion and perspectives}

311 The present dataset greatly expands previous compilations of EWL in squamate reptiles by nearly

312 doubling the number of species (ca. 100 species in Mautz, 1982; 139 species in Cox \& Cox, 2015)

313 It also provides exhaustive metadata about methods, contexts and protocols in which each unique

314 data point was obtained. This expanded and fully annotated dataset will ease transparent and reproducible statistical manipulation of EWL data for future studies, allow to examine how much

316 variation in EWL is caused by methodological factors instead of ecological or evolutionary drivers,

317 facilitate the estimation of skin resistance for an expanded list of species and will therefore ease 318 comparative analyses of EWL. The dataset may also assist in estimates of TEWL, CWL and RWL, 
the conversion between different measurement scales and the identification of records performed

320 within pre-defined set of conditions such as standard records with non-manipulated animals at rest.

321 This is particularly important given the substantial differences in methodology among studies and

322 the inherent variability in EWL values caused by air temperature, air humidity and air velocity

323 during measurements. Potential case studies will include methodological analyses of microclimatic

324 factors such as temperature and humidity, partitioning of intra- and inter-specific variation and

325 comparative phylogenetic analyses of the diversification and environmental constraints on EWL

326 across species.

Despite earlier suggestions to better standardize EWL measurement protocols (Mautz,

328 1982), the current data covers a broad range of methods and contexts, and we found it difficult to

329 suggest an optimal and unique way to measure EWL in squamate reptiles because the exact protocol

330 will always depend on the specific research questions. Obviously, broad-scale comparisons of EWL

331 in poorly explored taxa and geographic areas would benefit from the use of simple approaches

332 where water loss is measured with a gravimetric method on animals at rest during a few hours in

333 controlled conditions in a ventilated box (Garcia-Porta et al., 2019). On the other hand, functional

334 studies will require detailed quantification of the processes and patterns of water loss and therefore

335 continuous-time gas measurements following standard guidelines on animals at rest in controlled

336 conditions in flow-through chambers (Lighton, 2018).

337 We hope that the compiled metadata information of our database (see Figure 1B) will foster

338 the improvement of data reporting standards. In particular, we recommend that future studies of

339 EWL in squamate reptiles report systematically details of animal origin, husbandry conditions

340 before measurements, protocols and measurements conditions, and provide central tendencies and

341 dispersion statistics on absolute scales (mg per hour) or supply the raw data. We also hope to

342 stimulate future researchers to collect and report all the variables necessary to quantify skin

343 resistance to water loss $R_{s}$, a potentially central metric to mechanistic modeling approaches (Riddell 
et al., 2017). For example, macro-ecological studies may focus on spatial analyses of how climatic

345 and other abiotic aspects of the habitat interact to determine species distributions and their

346 vulnerability to environmental disturbances.

Our dataset further identifies critical geographic and taxonomic gaps that may be valuable in guiding future investigations. The limited geographic and poor taxonomic coverage of our dataset reflect known gaps in herpetological and ecological research (Roll et al., 2017; Meiri, 2018; Etard et al., 2020). Such gaps and biases are also not surprising for functional traits related to physiology for which coverage is much lower than ecological, life history, and morphological traits $(>20 \%$ species coverage according to Etard et al., 2020). Given that the water balance physiology of squamate reptiles has been far less investigated than their thermal biology, we sustain that it is crucial that a gap-filling effort be directed to the sampling of more data in undersampled areas and taxonomic groups. More specifically, we shall mention the scarcity of data for Gekkonidae, Asia. The database will be regularly updated with these new data to provide a central resource for ecological and evolutionary research on this particular animal group.

\section{Data Accessibility}

360 Data are available on the Global Ecology and Biogeography webpage and on Zenodo (doi: 361 10.5281/zenodo.3666172).

\section{References}

Addo-Bediako, A., Chown, S.L. \& Gaston, K.J. (2001) Revisiting water loss in insects: a large scale view. Journal of Insect Physiology, 47, 1377-1388.

Albright, T.P., Mutiibwa, D., Gerson, A.R., Smith, E.K., Talbot, W.A., O’Neill, J.J., McKechnie, A.E. \& Wolf, B.O. (2017) Mapping evaporative water loss in desert passerines reveals an expanding threat of lethal dehydration. Proceedings of the National Academy of Sciences, 114, 2283-2288. 
Barroso, F.M., Carretero, M.A., Silva, F. \& Sannolo, M. (2016) Assessing the reliability of thermography to infer internal body temperatures of lizards. Journal of Thermal Biology, 62, 90-96.

Bennett, J.M., Calosi, P., Clusella-Trullas, S., Martínez, B., Sunday, J., Algar, A.C., Araújo, M.B., Hawkins, B.A., Keith, S., Kühn, I., Rahbek, C., Rodríguez, L., Singer, A., Villalobos, F., Olalla-Tárraga, M.Á. \& Morales-Castilla, I. (2018) GlobTherm, a global database on thermal tolerances for aquatic and terrestrial organisms. Scientific Data, 5, 180022.

Boyle, W.A., Shogren, E.H. \& Brawn, J.D. (2020) Hygric Niches for Tropical Endotherms. Trends in Ecology \& Evolution, S0169534720301737.

Brischoux, F., Tingley, R., Shine, R. \& Lillywhite, H.B. (2012) Salinity influences the distribution of marine snakes: implications for evolutionary transitions to marine life. Ecography, 35, 994-1003.

Buckley, L.B., Hurlbert, A.H. \& Jetz, W. (2012) Broad-scale ecological implications of ectothermy and endothermy in changing environments. Global Ecology and Biogeography, 21, 873885.

Buckley, L.B., Rodda, G.H. \& Jetz, W. (2008) Thermal and energetic constraints on ectotherm abundance: a global test using lizards. Ecology, 89, 48-55.

Chase, J.M. \& Leibold, M.A. (2003) Ecological niches: linking classical and contemporary approaches, University of Chicago Press.

Chown, S.L., Sorensen, J.G. \& Terblanche, J.S. (2011) Water loss in insects: An environmental change perspective. Journal of Insect Physiology, 57, 1070-1084.

Cox, C.L. \& Cox, R.M. (2015) Evolutionary shifts in habitat aridity predict evaporative water loss across squamate reptiles. Evolution, 69, 2507-2516.

Díaz, S., Kattge, J., Cornelissen, J.H.C., Wright, I.J., Lavorel, S., Dray, S., Reu, B., Kleyer, M., Wirth, C., Colin Prentice, I., Garnier, E., Bönisch, G., Westoby, M., Poorter, H., Reich, P.B., Moles, A.T., Dickie, J., Gillison, A.N., Zanne, A.E., Chave, J., Joseph Wright, S., Sheremet'ev, S.N., Jactel, H., Baraloto, C., Cerabolini, B., Pierce, S., Shipley, B., Kirkup, D., Casanoves, F., Joswig, J.S., Günther, A., Falczuk, V., Rüger, N., Mahecha, M.D. \& Gorné, L.D. (2016) The global spectrum of plant form and function. Nature, 529, 167-171. Dillon, M.E., Wang, G. \& Huey, R.B. (2010) Global metabolic impacts of recent climate warming. Nature, 467, 704-U88. 
Dmi'el, R. (2001) Skin resistance to evaporative water loss in reptiles: a physiological adaptive mechanism to environmental stress or a phyletically dictated trait? Israel Journal of Zoology, 47, 56-67.

Dmi'el, R. (1998) Skin resistance to evaporative water loss in viperid snakes: habitat aridity versus taxonomic status. Comparative Biochemistry and Physiology Part A: Molecular \& Integrative Physiology, 121, 1-5.

Etard, A., Morrill, S. \& Newbold, T. (2020) Global gaps in trait data for terrestrial vertebrates. Global Ecology and Biogeography, 29, 2143-2158.

Garcia-Porta, J., Irisarri, I., Kirchner, M., Rodríguez, A., Kirchhof, S., Brown, J.L., MacLeod, A., Turner, A.P., Ahmadzadeh, F., Albaladejo, G., Crnobrnja-Isailovic, J., De la Riva, I., Fawzi, A., Galán, P., Göçmen, B., Harris, D.J., Jiménez-Robles, O., Joger, U., Jovanović Glavaš, O., Karış, M., Koziel, G., Künzel, S., Lyra, M., Miles, D., Nogales, M., Oğuz, M.A., Pafilis, P., Rancilhac, L., Rodríguez, N., Rodríguez Concepción, B., Sanchez, E., Salvi, D., Slimani, T., S’khifa, A., Qashqaei, A.T., Žagar, A., Lemmon, A., Moriarty Lemmon, E., Carretero, M.A., Carranza, S., Philippe, H., Sinervo, B., Müller, J., Vences, M. \& Wollenberg Valero, K.C. (2019) Environmental temperatures shape thermal physiology as well as diversification and genome-wide substitution rates in lizards. Nature Communications, 10, 4077.

Gates, D.M. (1980) Biophysical ecology, Springer-Verlag, New York, USA.

Gouveia, S.F., Bovo, R.P., Rubalcaba, J.G., Silva, F.R.D., Maciel, N.M., Andrade, D.V. \& Martinez, P.A. (2019) Biophysical Modeling of Water Economy Can Explain Geographic Gradient of Body Size in Anurans. The American Naturalist, 193, 51-58.

Kattan, G.H. \& Lillywhite, H.B. (1989) Humidity acclimation and skin permeability in the lizard Anolis carolinensis. Physiological Zoology, 62, 593-606.

Kearney, M. \& Porter, W. (2009) Mechanistic niche modelling: combining physiological and spatial data to predict species' ranges. Ecology Letters, 12, 334-350.

Kearney, M. \& Porter, W.P. (2004) Mapping the fundamental niche: Physiology, climate, and the distribution of a nocturnal lizard. Ecology, 85, 3119-3131.

Kearney, M., Shine, R. \& Porter, W.P. (2009) The potential for behavioral thermoregulation to buffer "cold-blooded" animals against climate warming. Proceedings of the National Academy of Sciences, 106, 3835-3840.

Kearney, M.R., Jusup, M., McGeoch, M.A., Kooijman, S.A.L.M. \& Chown, S.L. (2021) Where do functional traits come from? The role of theory and models. Functional Ecology, n/a. 
Kearney, M.R., Munns, S.L., Moore, D., Malishev, M. \& Bull, C.M. (2018) Field tests of a general ectotherm niche model show how water can limit lizard activity and distribution. Ecological Monographs.

Kearney, M.R. \& Porter, W.P. (2020) NicheMapR - an R package for biophysical modelling: the ectotherm and Dynamic Energy Budget models. Ecography, 43, 85-96.

Le Galliard, J.-F., Chabaud, C., de Andrade, D.O.V., Brischoux, F., Carretero, M.A., Dupoué, A., Gavira, R., Lourdais, O., Sannolo, M. \& van Dooren, T.J.M. (2020) SquamEWL: A worldwide and annotated database of evaporative water loss rates in squamate reptiles.

Lertzman-Lepofsky, G.F., Kissel, A.M., Sinervo, B. \& Palen, W.J. (2020) Water loss and temperature interact to compound amphibian vulnerability to climate change. Global Change Biology, 26, 4868-4879.

Lighton, J.R.B. (2018) Measuring Metabolic Rates: A Manual for Scientists, Second Edition. Oxford University Press, Oxford, New York.

Lillywhite, H.B. (2017) Feeding begets drinking: insights from intermittent feeding in snakes. Journal of Experimental Biology, 220, 3565-3570.

Loughran, C.L. \& Wolf, B.O. (2020) The functional significance of panting as a mechanism of thermoregulation and its relationship to the critical thermal maxima in lizards. Journal of Experimental Biology, 223.

Mautz, W.J. (1980) Factors influencing evaporative water loss in lizards. Comparative Biochemistry and Physiology Part A, 67A, 429-437.

Mautz, W.J. (1982) Patterns of evaporative water loss. Biology of the Reptilia (ed. by C. Gans) and F.H. Pough), pp. 443-481. Academic Press, London.

Meiri, S. (2018) Traits of lizards of the world: Variation around a successful evolutionary design. Global Ecology and Biogeography, 27, 1168-1172.

Moen, D.S., Winne, C.T. \& Reed, R.N. (2005) Habitat-mediated shifts and plasticity in the evaporative water loss rates of two congeneric pit vipers (Squamata, Viperidae, Agkistrodon). Evolutionary Ecology Research, 7, 759-766.

Noble, D.W.A., Stenhouse, V., Riley, J.L., Warner, D.A., While, G.M., Du, W.-G., Uller, T. \& Schwanz, L.E. (2018) A comprehensive database of thermal developmental plasticity in reptiles. Scientific Data, 5, 180138.

Oufiero, C.E. \& Van Sant, M.J. (2018) Variation and repeatability of cutaneous water loss and skin resistance in relation to temperature and diel variation in the lizard Sceloporus consobrinus. Journal of Comparative Physiology B, 188, 671-681. 
Pintor, A.F.V., Schwarzkopf, L. \& Krockenberger, A.K. (2016) Hydroregulation in a tropical dryskinned ectotherm. Oecologia, 182, 925-931.

Pirtle, E.I., Tracy, C.R. \& Kearney, M.R. (2019) Hydroregulation. Behavior of Lizards:

Evolutionary and Mechanistic Perspectives (ed. by V. Bels) and A.P. Russell), pp. 343-374.

CRC Press (Tayllor \& Francis Group), Boca Raton, Florida, USA.

R Core Team (2020) R: A Language and Environment for Statistical Computing, R Foundation for Statistical Computing, Vienna, Austria.

Riddell, E.A., Apanovitch, E.K., Odom, J.P. \& Sears, M.W. (2017) Physical calculations of resistance to water loss improve predictions of species range models. Ecological Monographs, 87, 21-33.

Riddell, E.A., Iknayan, K.J., Wolf, B.O., Sinervo, B. \& Beissinger, S.R. (2019) Cooling requirements fueled the collapse of a desert bird community from climate change. Proceedings of the National Academy of Sciences, 116, 21609-21615.

Roll, U., Feldman, A., Novosolov, M., Allison, A., Bauer, A.M., Bernard, R., Böhm, M., CastroHerrera, F., Chirio, L., Collen, B., Colli, G.R., Dabool, L., Das, I., Doan, T.M., Grismer, L.L., Hoogmoed, M., Itescu, Y., Kraus, F., LeBreton, M., Lewin, A., Martins, M., Maza, E., Meirte, D., Nagy, Z.T., de C Nogueira, C., Pauwels, O.S.G., Pincheira-Donoso, D., Powney, G.D., Sindaco, R., Tallowin, O.J.S., Torres-Carvajal, O., Trape, J.-F., Vidan, E., Uetz, P., Wagner, P., Wang, Y., Orme, C.D.L., Grenyer, R. \& Meiri, S. (2017) The global distribution of tetrapods reveals a need for targeted reptile conservation. Nature Ecology \& Evolution, 1, $1677-1682$.

Rozen-Rechels, D., Badiane, A., Agostini, S., Meylan, S. \& Le Galliard, J.-F. (2020) Water restriction induces behavioral fight but impairs thermoregulation in a dry-skinned ectotherm. Oikos, 129, 572-584.

Rozen-Rechels, D., Dupoué, A., Lourdais, O., Chamaillé-Jammes, S., Meylan, S., Clobert, J. \& Galliard, J.-F.L. (2019) When water interacts with temperature: Ecological and evolutionary implications of thermo-hydroregulation in terrestrial ectotherms. Ecology and Evolution, 9, 10029-10043.

Sannolo, M., Civantos, E., Martín, J. \& Carretero, M.A. (2020) Variation in field body temperature and total evaporative water loss along an environmental gradient in a diurnal ectotherm. Journal of Zoology, 310, 221-231. 
Schneider, F.D., Fichtmueller, D., Gossner, M.M., Güntsch, A., Jochum, M., König-Ries, B., Provost, G.L., Manning, P., Ostrowski, A., Penone, C. \& Simons, N.K. (2019) Towards an ecological trait-data standard. Methods in Ecology and Evolution, 10, 2006-2019.

Senzano, L.M. \& Andrade, D.V. (2018) Temperature and dehydration effects on metabolism, water uptake and the partitioning between respiratory and cutaneous evaporative water loss in a terrestrial toad. Journal of Experimental Biology, 221, jeb188482.

Sinervo, B., Mendez-de-la-Cruz, F., Miles, D.B., Heulin, B., Bastiaans, E., Cruz, M.V.S., LaraResendiz, R., Martinez-Mendez, N., Calderon-Espinosa, M.L., Meza-Lazaro, R.N., Gadsden, H., Avila, L.J., Morando, M., De la Riva, I.J., Sepulveda, P.V., Rocha, C.F.D., Ibargüengoytía, N., Puntriano, C.A., Massot, M., Lepetz, V., Oksanen, T.A., Chapple, D.G., Bauer, A.M., Branch, W.R., Clobert, J. \& Sites, J.W. (2010) Erosion of lizard diversity by climate change and altered thermal niches. Science, 328, 894-899.

Sunday, J.M., Bates, A.E. \& Dulvy, N.K. (2012) Thermal tolerance and the global redistribution of animals. Nature Climate Change, 2, 686-690.

Tattersall, G.J., Cadena, V. \& Skinner, M.C. (2006) Respiratory cooling and thermoregulatory coupling in reptiles. Respiratory Physiology \& Neurobiology, 154, 302-318.

Trabucco, A. \& Zomer, R. (2019) Global Aridity Index and Potential Evapotranspiration (ET0) Climate Database v2.

Uetz, P. \& Etzold, T. (1996) The EMBL/EBI Reptile Database. Herpetological Review, 27, 174175.

Van Sant, M.J., Oufiero, C.E., Muñoz-Garcia, A., Hammond, K.A. \& Williams, J.B. (2012) A phylogenetic approach to total evaporative water loss in mammals. Physiological and Biochemical Zoology: Ecological and Evolutionary Approaches, 85, 526-532.

Violle, C., Navas, M.-L., Vile, D., Kazakou, E., Fortunel, C., Hummel, I. \& Garnier, E. (2007) Let the concept of trait be functional! Oikos, 116, 882-892.

Warburg, M.R. (1965) The influence of ambient temperature and humidity on the body temperature and water loss from two Australian lizards, Tiliqua rugosa (Gray) (Scincidae) and Amphibolurus barbatus cuvier (Agamidae). Australian Journal of Zoology, 13, 331-350.

Zheng, Y. \& Wiens, J.J. (2016) Combining phylogenomic and supermatrix approaches, and a timecalibrated phylogeny for squamate reptiles (lizards and snakes) based on 52 genes and 4162 species. Molecular Phylogenetics and Evolution, 94, 537-547. 


\section{Figure legends}

364 Figure 1. Components of water loss in squamate reptiles and metadata required to describe evaporative water loss $(\mathrm{EWL})$. A. The total water budget of the animal rests on balance between water intake from food and drinking of rain, free standing water or moisture, metabolic water production and water loss from respiratory evaporation (RWL), water loss from skin, ocular or cloacal evaporation (CWL) and water loss from feces and excreta. Total evaporative water loss (TEWL) is the sum of total respiratory and cutaneous evaporative water loss and can be measured from post-absorptive animals in controlled laboratory conditions provided they do not produce feces or excreta. B. Controlled description of EWL rates data using metadata describing properties of entities (observations, including species, population and animals), observations

372 (including characteristics, protocols and context) and values. A rich metadata set is especially needed to 373 describe the methodology and the environmental conditions when EWL was measured. Each concept in this figure refers to one or several columns of metadata in our database (see Table 1 for details). The database was linked with associated data on metabolic rate (MR) whenever the study reported concurrent estimates of

376 MR for the same animals.

377 Figure 2. A. Geographic distribution of data records with exact coordinates of sampling location when 378 available. The geographic location of data records is mapped over a raster map of the Global Aridity Index 379 (GAI) for the 1970-2000 period (Trabucco \& Zomer, 2019). The aridity index represents the ratio between rainfall and a measure of potential evapo-transpiration (hyper-arid: GAI $<0.03$; arid: GAI $<0.2$; semi-arid: $0.2<\mathrm{GAI}<0.5$; dry sub-humid: $0.5<\mathrm{GAI}<0.65$; humid: GAI $>0.65$; the scale includes a larger range of humid conditions for the sake of visualization). B. Phylogenetic tree of the Squamata according to a recent time-calibrated phylogeny by (Zheng \& Wiens, 2016). We calculated the proportion of species sampled (black portion of each barplot) according to the Reptile Database (Uetz \& Etzold, 1996) and the total number of records for each family. Data deficient families are highlighted in blue. C. Allometric scaling of water loss rates (mg water loss per hour) with body mass in 1485 records across 305 species for which TEWL, total CWL and total RWL could be calculated. All individual data points are displayed on a log-log scale together with the best linear non-phylogenetic regression line for each EWL component. 


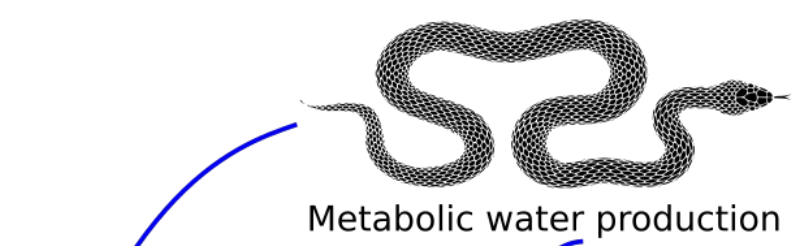

Total evaporative water loss (TEWL)

\section{B. Controlled description of EWL}

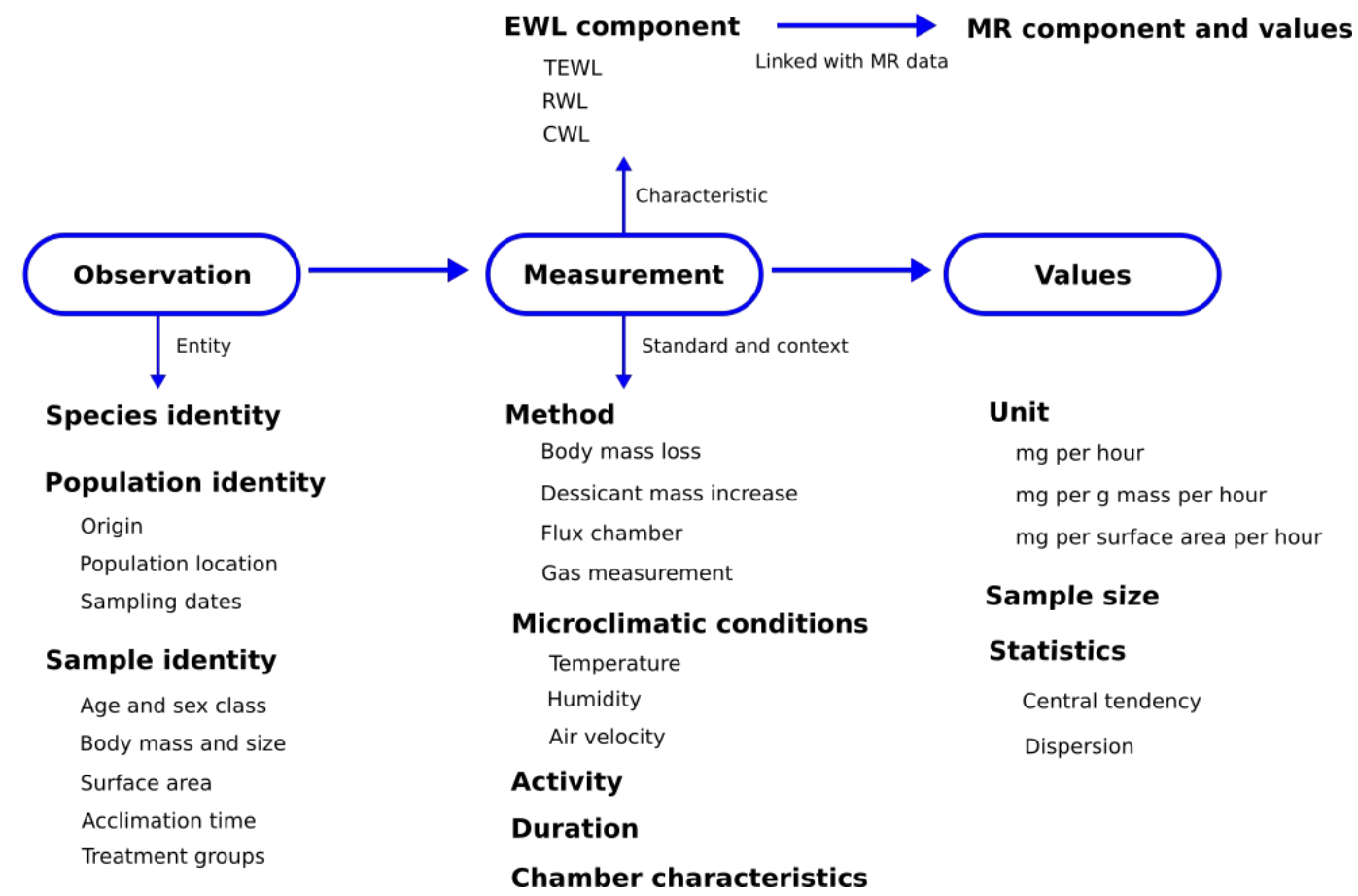



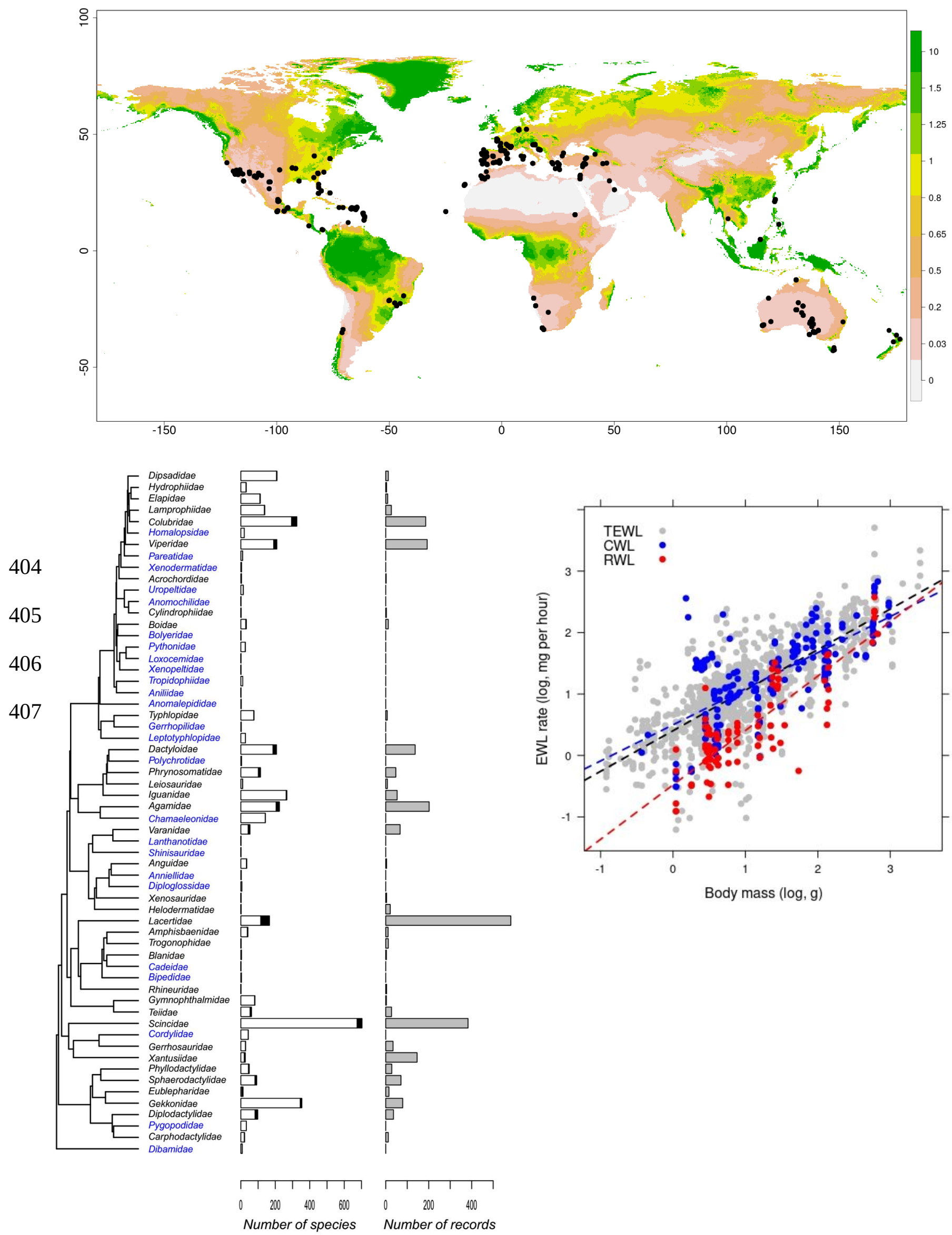